\title{
Wie gut ist die ärztliche Weiterbildung? Ärztekammern starten bundesweite Umfrage
}

Die Unzufriedenheit mit der ärztlichen Weiterbildung in Deutschland ist groß. Um Klarheit über die Stärken und Schwächen des Systems zu gewinnen, sollen Weiterbilder und Assistenten künftig regelmäßig befragt werden.

In den vergangenen Jahren wurde die Weiterbildungssituation in Deutschland häufig dafür verantwortlich gemacht, dass junge Ärztinnen und Ärzte aus der Patientenversorgung aussteigen, in andere Berufsfelder wechseln oder ins Ausland abwandern. Verlässliche Daten über den Grad der Unzufriedenheit und die Gründe, dem kurativen System schon in jungen Jahren den Rücken zu kehren, gibt es derzeit allerdings nicht.

\section{Startschuss fällt im Mai 2009}

Im Mai 2009 fällt daher erstmals der Startschuss zu einer bundesweiten Befragung: Bundesärztekammer und Landesärztekammern wollen in Erfahrung bringen, wie Weiterbildungsassistenten und Weiterbildungsbefugte die Facharzt-Weiterbildung in Deutschland bewerten. 16 Ärztekammern beteiligen sich an dem Projekt „Evaluation der Weiterbildung“.

Nur die Sächsische Landesärztekammer hat sich gegen eine Teilnahme entschieden. Dort habe man bereits im Jahr 2007 eine Befragung von Weiterbildungsassi-

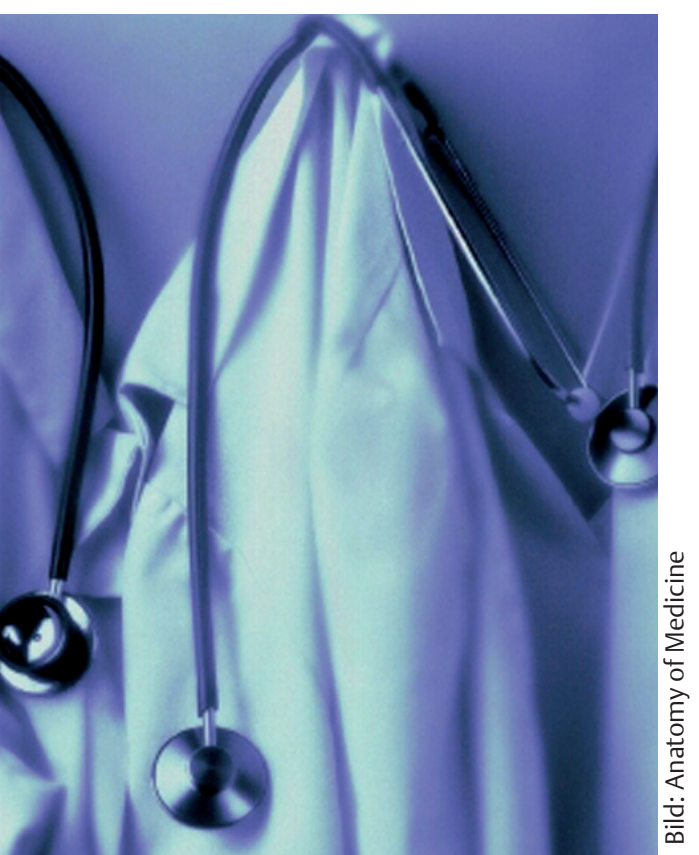

stenten durchgeführt - mit positiven Ergebnissen, heißt es.

\section{Evaluation u. a. der Lern-, Führungs- und Fehlerkultur}

Den Weiterbildungsassistenten und Weiterbildungsbefugten werden Fragen zu folgenden Aspekten der Weiterbildung gestellt: Vermittlung von Fachkompetenzen, Lernkultur, Führungskultur, Fehlerkultur/Patientensicherheit, Entscheidungskultur, Betriebskultur (Arbeitsklima der Weiterbildungsstätte) sowie Anwendung evidenzbasierter Medizin.

Alle im Zusammenhang mit dem Projekt erhobenen und verwendeten Daten werden gemäß den gesetzlichen Bestimmungen über den Datenschutz behandelt. Eine Rückspiegelung der Einzelfragebögen zur Ärztekammer ist ausgeschlossen. Rückschlüsse auf die Identität der Befragten sind nicht möglich.

\section{Befragung soll erstmals Ver-} gleichsmöglichkeiten schaffen Durch die Bewertung der einzelnen Weiterbildungsstätten und die Darstellung der Ergebnisse der Mittelwerte auf Bundes- und Landesebene soll die Befragung erstmals Vergleichsmöglichkeiten schaffen und Transparenz über die Weiterbildungssituation herstellen. „Die Umfrage dient dazu, die aktuelle Situation in den Weiterbildungsstätten in ihren positiven wie negativen Facetten darzustellen, um z. B. Handlungskonzepte für strukturierte Weiterbildungsabläufe zu erarbeiten. Mit den gewonnenen Erkenntnissen wollen wir Anreize entwickeln, um den Nachwuchs zu motivieren, in Deutschland als Arzt tätig zu werden. Denn nur gute Weiterbildungsbedingungen können der Abwanderung junger Ärztinnen und Ärzte in andere Berufsfelder oder ins Ausland entgegenwirken“, erklärt Dr. H. Hellmut Koch, Präsident der Bayerischen Landes- ärztekammer und Vorsitzender der Weiterbildungsgremien der Bundesärztekammer, die Projektziele.

\section{Ziel: Abwanderung in andere \\ Berufsfelder verhindern}

Wissenschaftlich ausgewertet werden die Daten durch die Eidgenössische Technische Hochschule Zürich (ETHZ), die seit 12 Jahren eine entsprechende Umfrage der Schweizer Ärztekammer begleitet.

Bei der Befragung wird eine einheitliche Erhebungsform und Befragungsmethodik zugrunde gelegt, wie sie in den Ärztekammern Hamburg und Bremen erstmals in den Jahren 2006/07 anhand standardisierter Fragebögen erfolgreich angewandt wurde. Die Befragungsergebnisse dort haben gezeigt, dass nur knapp die Hälfte der befragten Assistenten den Eindruck äußerte, dass eine gute Weiterbildung wichtiges oder wichtigstes Ziel der Abteilung sei.

Der Besuch von Weiterbildungsveranstaltungen findet bei der Mehrzahl außerhalb der Regelarbeitszeit statt. Die Kosten für den Besuch von Veranstaltungen werden manchmal teilweise, häufig aber gar nicht vom Arbeitgeber übernommen.

In der Regel arbeiten die Assistenten lediglich auf der Basis mündlicher oder gar nicht vereinbarter Lern- und Weiterbildungsziele. Über zwei Drittel der Assistenten gaben darüber hinaus an, bei Entscheidungen innerhalb des Krankenhauses, die ihre Weiterbildung betreffen, nicht ausreichend oder gar nicht informiert $\mathrm{zu}$ werden.

Dennoch haben fast $62 \%$ der Befragten ihre Weiterbildungsstätte mit nur leichten Einschränkungen oder aus voller Überzeugung weiterempfohlen. Ebenso viele sind mit ihrer Arbeitssituation einigermaßen bis sehr zufrieden. Die Mehrzahl der Weiterbildungsbefugten in Hamburg und Bremen waren der Meinung, dass eine gute Weiterbildung innerhalb der tariflichen Arbeitszeit so gut wie nicht mehr zu leisten ist. Größere Ressourcen, sowohl zeitlich wie finanziell, werden dringend für erforderlich gehalten. Klaus Schmidt, Planegg 